\title{
ZONEAMENTO CLIMÁTICO PARA TECA, CEDRO AUSTRALIANO, NIM INDIANO E PUPUNHA NO ESTADO DO ESPÍRITO SANTO
}

\author{
Valéria Hollunder Klippel ${ }^{1}$, José Eduardo Macedo Pezzopane ${ }^{2}$, José Ricardo Macedo Pezzopane ${ }^{3}$, \\ Roberto Avelino Cecílio ${ }^{4}$, Fábio da Silveira Castro ${ }^{5}$, Luciano Roncete Pimenta ${ }^{5}$ \\ ${ }^{1}$ Eng $^{\mathrm{a}}$. Florestal, M.Sc., Depto. de Eng. Agrícola, UFV, Viçosa, MG, Brasil - vhklippel@ gmail.com \\ ${ }^{2}$ Eng. Florestal, Dr., Centro de Ciências Agrárias, UFES, Alegre, ES, Brasil - pezzopane2007@yahoo.com.br \\ ${ }^{3}$ Eng. Agrônomo, Dr., Centro de Pesquisa de Pecuária do Sudeste, EMBRAPA, Alegre, ES, Brasil - jricardo@ cppse.embrapa.br \\ ${ }^{4}$ Eng. Agrícola, Dr., Centro de Ciências Agrárias. UFES, Alegre, ES, Brasil - racecilio@ gmail.com \\ ${ }^{5}$ Eng. Agrônomo, M.Sc., Depto. de Eng. Agrícola, UFV, Viçosa, MG, Brasil - fabiosilveira_70@ hotmail.com; \\ lucianorpimenta@yahoo.com.br \\ Recebido para publicação: 22/02/2013 - Aceito para publicação: 10/09/2013
}

\begin{abstract}
Resumo
Objetivou-se com este estudo realizar um zoneamento climático para as espécies Tectona grandis, Toona ciliata, Bactris gasipaes e Azadirachta indica, no estado do Espírito Santo. Utilizaram-se dados meteorológicos do período de 1977 a 2006, provenientes de 80 postos pluviométricos da ANA localizados dentro do estado e em 16 postos circunvizinhos, de 11 estações meteorológicas pertencentes ao INCAPER e de 3 do INMET. Os elementos climáticos usados foram a temperatura do ar e a deficiência hídrica anual para a capacidade de água disponível de $300 \mathrm{~mm}$. Fazendo-se uso de um sistema de informação geográfica, classificou-se o estado em zonas de aptidão climática. A espécie Azadirachta indica pode ser implantada em 54,7\% da área total, e esse valor elevado foi devido à baixa restrição em relação às exigências climáticas, quando comparado às outras espécies. Todavia, a Bactris gasipaes é a espécie que apresenta a menor porção de área apta para a sua implantação $(4,2 \%)$, devido às suas restrições hídricas e térmicas, que não condizem com as condições do estado. Tectona grandis e Toona ciliata apresentaram aptidão climática, respectivamente, em $33,3 \%$ e $27 \%$ da área total.

Palavra-chave: Espécies florestais; balanço hídrico; sistema de informação geográfica; aptidão climática.
\end{abstract}

\begin{abstract}
Climatic zoning for teca, australian cedar, neem and pupunha in state of Espirito Santo. This research aims to make a climatic zoning for the species: Tectona grandis, Toona ciliata, Bactris gasipaes and Azadirachta indica, in State of Espirito Santo. It was used meteorological data from 1977 to 2006, considering eighty rainfall posts of the ANA, located within the state and sixteen posts in surrounding states. Eleven weather stations belonged to INCAPER and three other stations to INMET. The climate elements used were air temperature and annual water deficiency, calculated for a soil water storage capacity of $300 \mathrm{~mm}$. It used geographical information system, ranking the State in areas of climatic suitability. As result, it indicates that the Azadirachta indica can be implanted in $54.7 \%$ of the total area of the State, this high value is due to its low restriction in relation to climatic requirements compared to other species. However, Bactris gasipaes is the species that has the lowest amount of area suitable for its implementation (4.2\%), due to its thermal and water restrictions not suitable to the State conditions. Tectona grandis and Toona ciliata revealed climatic suitability, respectively, in $33.3 \%$ and $27 \%$ of the total area of the State.

Keywords: Forest species; water balance; geographic information system; zoning; climate aptitude.
\end{abstract}

\section{INTRODUÇÃO}

A implantação de florestas comerciais é importante tanto para diminuir o déficit florestal quanto para a proteção dos remanescentes nativos e para a fixação de carbono (SOUZA et al., 2010; RIBEIRO et al., 2011; FERREIRA et al., 2012). 
É cada vez maior a busca por espécies com potencial florestal, buscando atender às tendências de mercado, maior produtividade e qualidade de sítios, entre outros aspectos (NAPPO et al., 2005). Segundo Tonello et al. (2008), 85\% de todos os produtos de origem florestal encontrados no mercado são originados de florestas plantadas.

A teca (Tectona grandis L. F.) é uma espécie que apresenta rápido crescimento e produz madeira de boa qualidade, com grande aceitação em todo o mundo (LORENZI et al., 2003), sendo utilizada na carpintaria, marcenaria, produção de peças de usos nobres e de móveis finos e, principalmente, na indústria da construção naval, onde é praticamente insubstituível, pelo fato de resistir ao sol, ao calor, ao frio e à água da chuva e do mar (RONDON NETO et al., 1998).

Outra espécie que exibe rápido crescimento é o cedro australiano (Toona ciliata M. Roem.) (LORENZI et al., 2003). Sua madeira é considerada uma das melhores da Austrália, apresentando boa durabilidade, fácil secagem e desdobro (PINHEIRO et al., 2003). Por ser considerada de alta qualidade, deve ser destinada preferencialmente a produtos de alto valor agregado, como, por exemplo, móveis finos e instrumentos musicais (SÁ et al., 2010).

Das folhas e óleo da Azadirachta indica A. Juss. (nim indiano), obtêm-se a azadirachtina, uma substância com propriedades inseticidas (LORENZI et al., 2003). Os inseticidas à base de nim são de baixo custo e podem ser produzidos de forma bastante simples. Além disso, são considerados menos poluentes, com baixo poder residual, oferecendo menor risco de intoxicação para mamíferos e aves (QUINTELA; PINHEIRO, 2004). O extrato bruto ou óleo extraído das folhas, caules e sementes também podem ter uso medicinal e na indústria cosmética (MARTINS et al., 2010).

O cultivo da Bactris gasipaes Kunth (pupunha) para produção de palmito vem despertando, desde a década de 70 , o interesse de agricultores de todo o País, principalmente devido à demanda elevada de palmito de boa qualidade, tanto interna quanto externa, e à alta lucratividade do setor (BOVI, 2000). A pupunheira é ideal para ser explorada em plantios comerciais, já que apresenta características desejáveis, como precocidade, perfilhamento, rendimento e qualidade do seu palmito (CHAIMSOHN, 2000), além de ser uma alternativa para produção sustentável dessa matéria-prima.

Características como condições naturais favoráveis, desenvolvimento tecnológico avançado da silvicultura e outras condições privilegiadas (localização geográfica, infraestrutura, logística de transporte e diversificação de plantas industriais) favorecem o crescimento da atividade florestal no estado do Espírito Santo (VALVERDE et al., 2005). Assim sendo, o zoneamento climático é uma importante ferramenta na delimitação de áreas com aptidões climáticas para a implantação e desenvolvimento de espécies florestais no estado, visto que estabelece indicadores térmicos e hídricos da região, além de auxiliar na tomada de decisões que podem trazer benefícios diretos para a cultura.

Diante do exposto, o objetivo principal deste estudo foi realizar o zoneamento climático de quatro espécies florestais no Espírito Santo: Tectona grandis (teca), Toona ciliata (cedro australiano), Bactris gasipaes (pupunha) e Azadirachta indica (nim indiano).

\section{MATERIAIS E MÉTODOS}

A área de estudo abrange o estado do Espírito Santo, situado geograficamente entre os meridianos $39^{\circ} 38^{\prime}$ e $41^{\circ} 50^{\prime}$ de longitude oeste e entre os paralelos $17^{\circ} 52^{\prime}$ e $21^{\circ} 19^{\prime}$ de latitude sul, sendo o clima influenciado de maneira marcante pela variação de altitude. Conforme o sistema de classificação de Köppen, a região enquadra-se nas zonas climáticas A e C, que identificam climas úmidos. No estado são encontrados os subtipos climáticos $\mathrm{Aw}, \mathrm{Am}, \mathrm{Cf}$ e $\mathrm{Cw}$, e também as variações $\mathrm{Cfa}, \mathrm{Cfb}$, Cwa e $\mathrm{Cwb}$ (SIQUEIRA et al., 2004).

Para caracterização da precipitação pluviométrica no estado, foram utilizadas séries históricas (com dados de 30 anos) englobando um mesmo período (1977-2006), coletadas em 11 estações meteorológicas do Instituto Capixaba de Pesquisa e Extensão Agropecuária (INCAPER) e em 3 estações do Instituto Nacional de Meteorologia (INMET), além de 80 postos pluviométricos da Agência Nacional de Águas (ANA) no Espírito Santo e 16 postos pluviométricos também pertencentes à ANA localizados fora do estado, tendo como objetivo minimizar o efeito de borda no processo de interpolação, assim como realizado por Andrade (1998), Acosta (1997), Moreira (1997) e Castro (2008). No total, foram utilizados 110 pontos de medição (Figura 1). 
Como nos postos pluviométricos da ANA não são realizadas medidas de temperatura do ar, essa variável foi estimada em função da altitude, latitude e longitude do estado, através de equações de regressão múltipla desenvolvidas por Castro (2008). A temperatura do ar foi espacializada de acordo com as equações de regressão múltipla desenvolvidas por Castro (2008), aplicando-as à imagem digital de latitude, longitude e altitude do Espírito Santo, em um sistema de informações geográficas. Para os dados de altitude, utilizou-se um modelo digital de elevação, obtido através de dados de radar SRTM (Shuttle Radar Topography Mission).

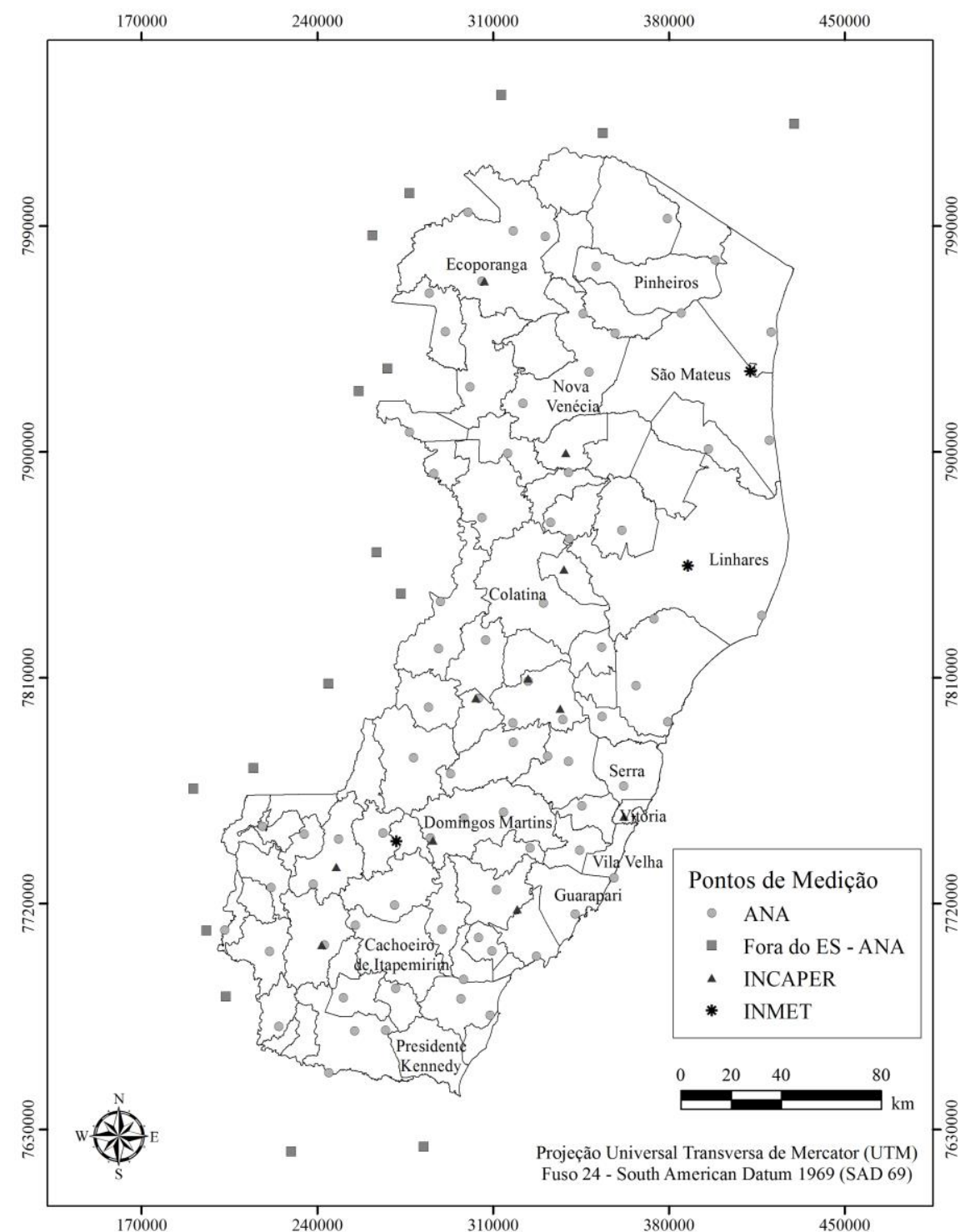

Figura 1. Distribuição espacial dos pontos de medição dos dados meteorológicos localizados no Espírito Santo e estados vizinhos.

Figure 1. Spatial distribution of the measuring climate data points located in state of Espirito Santo and surrounding states.

Mediante os dados meteorológicos de temperatura média do ar e precipitação pluviométrica para todas as localidades, foi calculado o balanço hídrico climático mensal, segundo Thornthwaite e Mather (1955), para os pontos de medidas selecionados no estudo, com o auxílio do programa "BHnorm", elaborado em planilha EXCEL por Rolim et al. (1998), utilizando uma capacidade máxima de 
armazenamento de água no solo (CAD) de $300 \mathrm{~mm}$, conforme é indicado para espécies florestais (PEREIRA et al., 2002). A evapotranspiração potencial foi calculada pelo modelo proposto por Thornthwaite (1948). Com o resultado do balanço hídrico em cada posto pluviométrico, foi realizada a interpolação dos dados de deficiência hídrica utilizando-se o método de krigagen modelo esférico, como indicado por Castro et al. (2010a) e utilizado por Castro et al. (2010b), permitindo, assim, a elaboração da caracterização digital da disponibilidade hídrica no estado.

As imagens Raster SRTM apresentavam-se no formato GEOTIFF (16 bits) e foram mosaicadas em resolução espacial de $90 \mathrm{~m}$, projeção geográfica e datum horizontal WGS 84, utilizando-se o software ArcGis/ArcMap, através do módulo "Data Management Tools". O mosaico foi necessário para a obtenção de apenas uma única imagem com valores médios de altitude e suas respectivas coordenadas geográficas para toda a área em estudo. Como os dados da imagem do mosaico ainda estavam em suas condições originais, ou seja, projeção geográfica e elevações referenciadas para o geoide WGS 84 (World Geodetic System of 1984), foi preciso convertê-la para projeção UTM (Universal Transversa de Mercator) zona $24 \mathrm{~s}$, fuso que compreende todo o estado e datum referenciado para SAD-69 (South American Datum 1969), sistema de referência geodésico que é adotado para a América do Sul.

O zoneamento climático consistiu essencialmente na delimitação de áreas com aptidão climática para o cultivo das espécies estudadas, na qual estão estabelecidas as condições hídrico-termais ideais para o seu desenvolvimento e consequente produtividade. No que se refere aos índices térmicos e hídricos exigidos pela espécie para o seu desenvolvimento, eles foram classificados de acordo com as necessidades de cada uma, dividindo-se em duas classes de aptidão climática: apta e inapta. Foram consideradas "aptas" aquelas áreas cujas condições térmicas e hídricas apresentaram-se favoráveis para o bom desenvolvimento e produção da espécie em escala comercial. A área foi considerada "inapta" quando as características normais do clima não se apresentaram adequadas à exploração econômica da espécie, devido a limitações severas dos fatores hídricos ou térmicos, ou ambos, com marcante repercussão em sua produção, exigindo para que sejam corrigidas práticas agrícolas dispendiosas.

Com base nos estudos realizados pelo Instituto de Pesquisa e Estudos Florestais (IPEF, 2008), Campos (2007), Bergo e Lunz (2000) e Neves et al. (2003), foram estabelecidos os parâmetros térmicos e hídricos para as espécies em estudo (Tabela 1).

Tabela 1. Faixa de aptidão térmica e hídrica para as espécies em estudo.

Table 1. Range of thermal and water aptitude for the focused species.

\begin{tabular}{lcccc}
\hline \multirow{2}{*}{ Espécie } & \multicolumn{4}{c}{ Regiões } \\
\cline { 2 - 5 } & Aptas & Inaptas & Aptas & Inaptas \\
\cline { 2 - 5 } & Temperatura média anual (Ta) ${ }^{\circ} \mathbf{C}$ & Deficiência hídrica anual mm \\
\hline Tectona grandis & $\geq 22$ & $<22$ & $\leq 150$ & $>150$ \\
Toona ciliata & $19 \leq \mathrm{Ta} \leq 23$ & $<19 \mathrm{ou}>23$ & $\leq 100$ & $>100$ \\
Bactris gasipaes & $\geq 22,0$ & $<22,0$ & $\leq 50$ & $>50$ \\
Azadirachta indica & $\geq 21,0$ & $<21,0$ & $\leq 200$ & $>200$ \\
\hline
\end{tabular}

Definidos seus índices climáticos, utilizou-se o software ArcGis 9.2 / ArcMap® para realizar os cruzamentos e gerar todos os mapas digitais. Primeiramente realizou-se o mosaico das imagens SRTM, gerando uma imagem "raster" da altitude para o Espírito Santo, e a partir das coordenadas geográficas do limite do estado realizou-se uma interpolação linear através do módulo "ArcToolbox - Spatial Analyst Tools - Interpolation", obtendo-se assim as imagens "raster" de latitude e longitude.

De posse do mapa de temperatura média anual do ar, procedeu-se, através do módulo "ArcToolbox - Spatial Analyst Tools - Reclass", à sua reclassificação, considerando as faixas de aptidão conforme os índices térmicos estabelecidos para cada espécie na tabela 1.

De acordo com os dados de deficiência hídrica do solo, obtidos através do balanço hídrico climatológico (THORNTHWAITE; MATHER, 1955), realizou-se a espacialização desses valores, estipulando as áreas com deficiência hídrica para todo o estado. Para isso, procedeu-se à interpolação dos dados utilizando-se rotinas específicas de um SIG implementado através do software ArcGis 9.2 / ArcMap ${ }^{\circledR}$ pelo módulo "ArcToolbox - Spatial Analyst Tools - Interpolation", o qual deu origem ao mapa temático que representa as condições de deficiência hídrica do estado. 
Uma vez estabelecido o mapa de classes de deficiência hídrica anual para o Espírito Santo, foram gerados os mapas de zonas de deficiência hídrica anual para cada espécie, de acordo com as suas faixas de aptidão hídrica, por intermédio da reclassificação através do módulo "Spatial Analyst Tools Reclass".

Finalmente, os mapas de zoneamento climático para as quatro espécies em estudo foram obtidos através do cruzamento dos mapas de temperatura média anual e deficiência hídrica anual já reclassificados, utilizando-se o software ArcGis 9.2 / ArcMap ${ }^{\circledR}$ pelo módulo "Spatial Analyst - Raster Calculator", conforme fluxograma da figura 2.

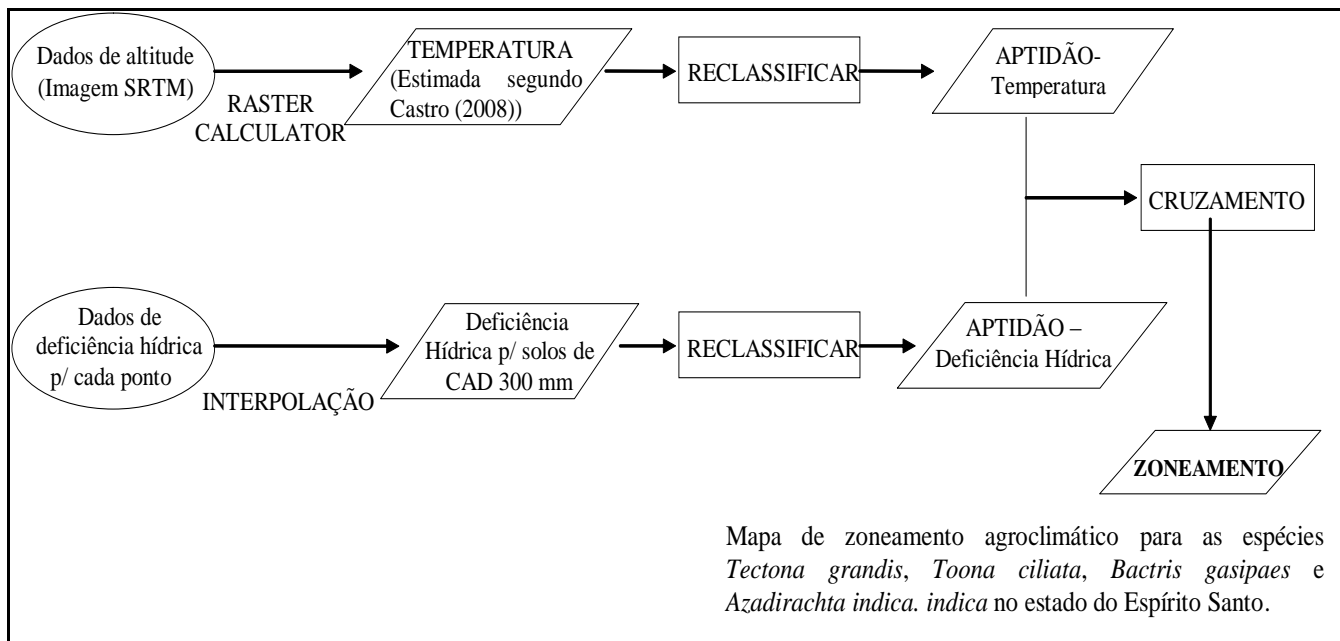

CAD: Capacidade de armazenamento disponível / storage available capacity

Figura 2. Fluxograma das etapas necessárias para obtenção dos mapas de zoneamento climático das espécies Tectona grandis, Toona ciliata, Bactris gasipaes e Azadirachta indica no Estado do Espírito Santo.

Figure 2. Flowchart of necessary stages in order to obtain maps of climatic zoning of Tectona grandis, Toona ciliata, Bactris gasipaes and Azadirachta indica in State of Espirito Santo.

\section{RESULTADOS E DISCUSSÃO}

De acordo com a figura 3, quase toda a região norte, a faixa litorânea e o vale do Rio Itapemirim ao sul do estado do Espírito Santo apresentam temperatura média anual acima de $24{ }^{\circ} \mathrm{C}$, enquanto a região serrana apresenta, em sua maior parte, temperatura média anual entre 18 e $22{ }^{\circ} \mathrm{C}$.

A região norte possui uma área maior de deficiência hídrica quando comparada com as regiões sul e serrana, onde os locais mais elevados apresentam deficiência hídrica anual abaixo de $50 \mathrm{~mm}$ (Figura 3). No extremo norte, a deficiência hídrica anual é superior a $250 \mathrm{~mm}$, e na região oeste, no Vale do Rio Doce, foram encontrados os maiores valores anuais de deficiência hídrica, com média próxima a $400 \mathrm{~mm}$, resultante da baixa disponibilidade hídrica e da alta demanda evaporativa.

Nota-se, na figura 4, que existem, em todo o estado do Espírito Santo, áreas potenciais com aptidão climática de implantação de povoamentos florestais para as espécies estudadas, de acordo com as bases estabelecidas para esse zoneamento.

Ainda de acordo com a figura 4, observa-se que, para o zoneamento climático da espécie Tectona grandis, o estado apresenta 33,3\% de áreas aptas, sendo que a maior parte encontra-se na região norte. As áreas inaptas totalizaram $66,7 \%$ de todo o território do estado. O principal fator de restrição para a implantação da cultura foi a deficiência hídrica, que encontra valores superiores a $150 \mathrm{~mm} / \mathrm{ano}$. A região centro-serrana, mesmo com o maior volume de chuvas e consequentemente menores valores de deficiência hídrica anual, possui temperaturas médias anuais entre 18 e $22{ }^{\circ} \mathrm{C}$, desfavorecendo o desenvolvimento da espécie. A maior parte das áreas aptas ao cultivo da teca no estado se encontram na região litorânea, onde são encontrados altos valores de temperatura média exigidos pela espécie, como é o 
caso dos municípios de Jaguaré, Sooretama, Linhares, Aracruz, João Neiva, Ibiraçu, Fundão, Serra, Cariacica, Vitória, Viana e São Mateus.

A espécie Toona ciliata apresentou $27 \%$ de áreas aptas e, consequentemente, $73 \%$ de áreas inaptas no estado do Espírito Santo (Figura 4). Na região norte do estado, apenas uma parte dos municípios de Ecoporanga, Barra de São Francisco e Água Doce do Norte apresentaram áreas climatologicamente aptas ao desenvolvimento da Toona ciliata, haja vista que essa região apresenta valores elevados de temperatura e deficiência hídrica. As regiões sul e serrana do estado são mais indicadas para o plantio dessa espécie, por apresentarem maior volume de chuva anual e, consequentemente, menores valores de deficiência hídrica, além de menores temperaturas médias. Esse resultado corrobora o estudo realizado por Sá et al. (2010), que utilizaram madeira de cedro australiano com 18 anos de idade proveniente do município de Marechal Floriano (ES), em um estudo da viabilidade técnica de produção de painéis cimento-madeira com resíduos da madeira dessa espécie. De acordo com Souza et al. (2010), a Toona ciliata é moderadamente tolerante à falta de água, mas altamente responsiva à quantidade de água disponibilizada durante o seu ciclo, com incrementos acentuados e rápidos.

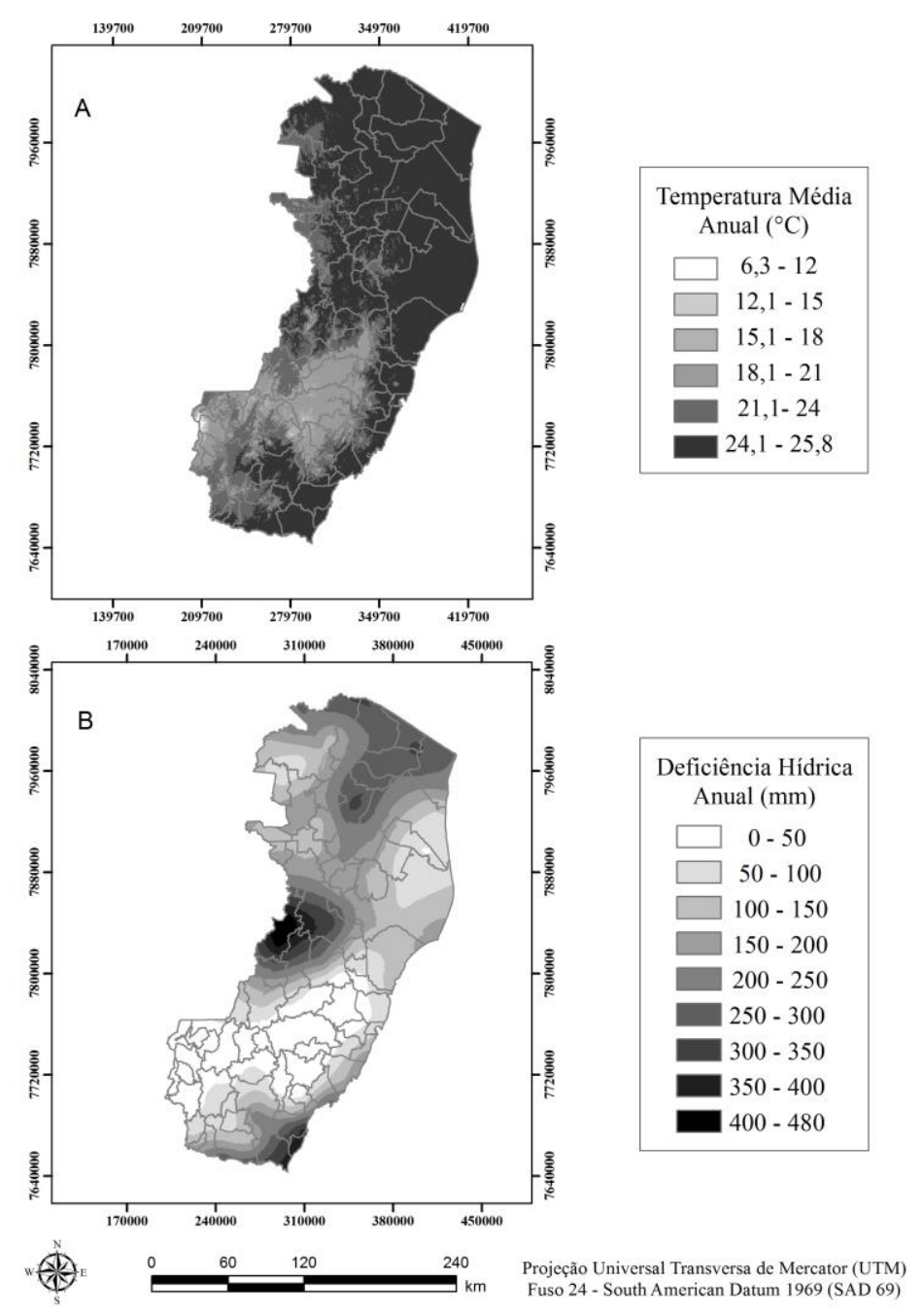

Figura 3. Espacialização da temperatura média anual do ar (A) e da deficiência hídrica anual acumulada (B) para o estado do Espírito Santo.

Figure 3. Spacialization of the air temperature annual average (A) and annual water deficit accumulated (B) for State of Espirito Santo. 

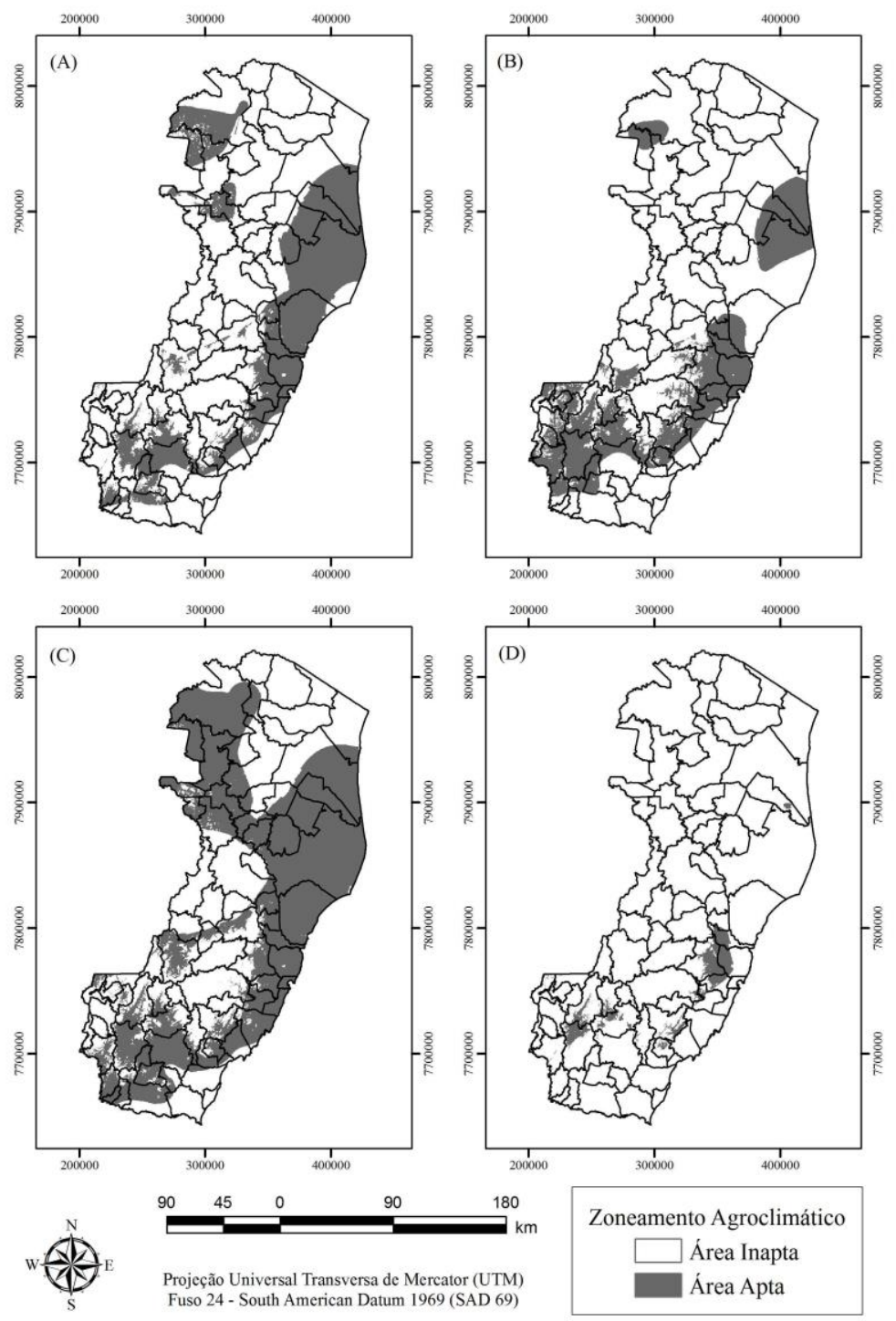

000

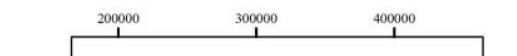

Figura 4. Zoneamento climático para: A - Tectona grandis; B - Toona ciliata; C - Azadirachta indica; D - Bactris gasipaes, no estado do Espírito Santo.

Figure 4. Climatic zoning for the species: A - Tectona grandis; B - Toona ciliate; C - Azadirachta indica; D - Bactris gasipaes, in State of Espirito Santo.

A Azadirachta indica é uma espécie de clima tropical, sendo resistente a longos períodos secos e tolerante a altas temperaturas (KOCH, 1990; NEVES et al., 2003). Conforme as exigências climáticas da espécie, mais da metade da área do estado do Espírito Santo (54,7\%) é apta para o seu plantio (Figura 4). Grande parte das áreas aptas estão localizadas no litoral e norte do estado, onde as temperaturas médias anuais são mais altas. No extremo norte do estado, onde a deficiência hídrica anual é superior a $250 \mathrm{~mm}$, e nas regiões sul e serrana, onde os valores de temperatura são mais baixos, as áreas inaptas para o cultivo dessa espécie totalizam $45,3 \%$. O nim é uma espécie vegetal extremamente plástica, pois sua regeneração natural ocorre na faixa de precipitação entre 400 e $700 \mathrm{~mm}$ (DELWAULLE, 1979), se adaptando às condições de solos degradados das principais regiões áridas do mundo (KOUL, 2004).

A espacialização do zoneamento climático para a implantação da Bactris gasipaes em todo o estado do Espírito Santo é apresentado na figura 4. As áreas climáticas aptas para essa cultura 
compreendem $4,2 \%$ do território, enquanto que a maior parte foi considerada inapta. Nota-se, então, que a pupunha não é indicada para implantação em grande parte do estado, pois as regiões com deficiência hídrica abaixo de $50 \mathrm{~mm}$ apresentam temperaturas médias anuais menores que $22^{\circ} \mathrm{C}$. Nas regiões onde a temperatura não é o fator de restrição, como no norte do estado, por exemplo, a deficiência hídrica está acima do suportado pela espécie. Nessas regiões, deve ser estudada a possibilidade de realização de irrigação, para que a cultura da pupunha possa ser implantada.

\section{CONCLUSÕES}

- O estado do Espírito Santo apresentou áreas com aptidão climática para todas as espécies estudadas, de acordo com as bases estabelecidas para este zoneamento.

- O nim indiano foi a espécie que melhor se adaptou às condições climáticas do estado, podendo ser implantada em mais da metade da área do mesmo.

- As áreas propícias para a implantação da teca e do cedro australiano são quase similares, principalmente no litoral e na região sul do estado. Porém a área com aptidão para a teca é mais abrangente, uma vez que essa espécie se desenvolve em locais com temperaturas mais elevadas e é mais tolerante ao déficit hídrico.

- Pelo fato de a pupunha apresentar parâmetros térmicos e hídricos muito distintos das condições climáticas encontradas no estado, essa espécie foi a que apresentou menor aptidão para implantação no estado do Espírito Santo.

- É bom ressaltar que este zoneamento levou em consideração apenas os fatores climáticos, sendo que, para uma agricultura produtiva, rentável e socioeconomicamente viável, o planejamento e as práticas de manejo adequadas são fundamentais.

\section{REFERÊNCIAS}

ACOSTA, V. H. Classificação ecológica do território brasileiro situado ao sul do paralelo $24^{\circ} \mathrm{S}$ uma abordagem climática. 86 p. Dissertação (Mestrado em Ciência Florestal) - Universidade Federal de Viçosa. Viçosa, 1997.

ANDRADE, L. A. Classificação ecológica do território brasileiro situado a leste do meridiano de $\mathbf{4 4}^{\circ}$ oeste e ao norte do paralelo de $\mathbf{1 6}^{\circ}$ sul: uma abordagem climática. 147 p. Tese (Doutorado em Ciências Florestais) - Universidade Federal de Viçosa. Viçosa, 1998.

BERGO, C. L.; LUNZ, A. M. P. Cultivo da pupunha para palmito no Acre. Embrapa, Circular Técnica n. 31, 2000.

BOVI, M. L. A. O agronegócio palmito de pupunha. O Agronômico, Campinas, v. 52, n. 1, 2000.

CAMPOS, V. M. C. de. Plantio de cedro australiano. Fundação Centro Tecnológico de Minas Gerais CETEC, Serviço Brasileiro de Respostas Técnicas, 2007.

CASTRO, F. da S. Zoneamento agroclimático para a cultura do pinus no estado do Espírito Santo. 121 f. Dissertação (Mestrado em Agrometeorologia Agrícola) - Universidade Federal do Espírito Santo, Alegre, ES, 2008.

CASTRO, F. da S.; PEZZOPANE, J. E. M.; CECÍlio, R. A.; PEZZOPANE, J. R. M.; XAVIER, A. C. Avaliação do desempenho dos diferentes métodos de interpoladores para parâmetros do balanço hídrico climatológico. Revista Brasileira de Engenharia Agrícola e Ambiental, v. 14, n. 8, p. 871-880, 2010.

CASTRO, F. da. S.; PEZZOPANE, J. E. M.; PEZZOPANE, J. R. M.; CECÍliO, R. A.; XAVIER, A. C. Zoneamento agroclimático para espécies do gênero Pinus no estado do Espírito Santo. Floresta, Curitiba, PR, v. 40, n. 1, p. 235-250, 2010.

CHAIMSOHN, F. P. Cultivo de pupunha e produção de palmito. Viçosa: Aprenda Fácil, 2000. 121 p.

DELWAULLE, J. C. Plantations forestières en Afrique Tropicale Sèche. Bois For Trop, v. 183: p. 3-18, 1979. 
NEVES, B. P. das; OLIVEIRA, I. P. de; NOGUEIRA, J. C. M. Cultivo e utilização do nim indiano. Embrapa Arroz e Feijão. Circular Técnica, 62, 2003.

FERREIRA, D. de A.; BARROSO, D. G.; SILVA, M. P. S. da; SOUZA, J. S. de; FREITAS, T. A. S. de; CARNEIRO, J. G. de. A. Influência da posição das miniestacas na qualidade de mudas de cedro australiano e no seu desempenho inicial no pós-plantio. Ciência Florestal, Santa Maria, v. 22, n. 4, p. 715-723, 2012.

INSTITUTO DE PESQUISA DE ESPÉCIES FLORESTAIS (IPEF). Disponível em: <http://www.ipef.br/ identificacao/tectona.grandis.asp>. Acesso em: 24/08/08.

KOCH, C. K. El arbor de la India (Azadirachta indica) y su utilizacion potential en el Equador con especial referencia a las propriedades plaguicidas de jus extratos. Equador: Convênio GTZ/MAG. 15 p., 1990.

KOUL, O. Neem: a global perspective. In KOUL, O., WAHAB, S. (Eds). Neem: today and in the new millennium. Dordrecht, Kluwer Academic Publishers, 2004, p. 1-19.

LORENZI, H.; SOUZA, H. M. de; TORRES, M. A. V.; BACHER, L. B. Árvores exóticas no Brasil: madeireiras, ornamentais e aromáticas. Nova Odessa, SP: Instituto Plantarum, 2003. 382 p.

MARTINS, M. de O.; NOGUEIRA, R. J. M. C.; AZEVEDO NETO, A. D. de; SANTOS, M. G. dos. Crescimento de plantas jovens de nim-indiano (Azadirachta indica A. Juss. - Meliaceae) sob diferentes regimes hídricos. Revista Árvore, Viçosa, MG, v. 34, n. 5, p. 771-779, 2010.

MOREIRA, I. P. S. Classificação ecológica do território brasileiro situado entre 16 e $24^{\circ}$ de latitude sul e 39 ${ }^{\circ} 51$ ' de longitude oeste. 156 p. Tese (Doutorado em Ciências Florestais) - Universidade Federal de Viçosa. Viçosa, 1997.

NAPPO, M. E.; NAPPO, A. E.; PAIVA, H. N. Zoneamento ecológico de pequena escala para nove espécies arbóreas de interesse florestal no estado de Minas Gerais. Revista Científica Eletrônica de Engenharia Florestal, v. 5. 14 p. 2005.

NEVES. B. P.; OLIVEIRA, I. P.; NOGUEIRA, J. C. M. Cultivo e utilização do nim indiano. Santo Antonio de Goiás: EMBRAPA, CNPAF, 2003. 12 p. Circular Técnica, 62.

PEREIRA, A. R.; ANGELOCCI, L. R.; SENTELHAS, P. C. Agrometeorologia: fundamentos e aplicações práticas. Guaíba: Agropecuária, 2002. 478 p.

PINHEIRO, A. L.; LANI, J. L.; COUTO, L. Cedro australiano cultivo e utilização. Viçosa: UFV, 2003. $42 \mathrm{p}$.

QUINTELA, E. D.; PINHEIRO, P. V. Efeito de extratos botânicos sobre a oviposição de Bemisia tabaci biótipo B em feijoeiro. Comunicado Técnico 92, Embrapa Arroz e Feijão, Santo Antônio de Goiás, GO, 6 p. 2004.

RIBEIRO, A. de O.; MORI, F. A.; MENDES, L. M. Características das dimensões das fibras e análise do ângulo microfibrilar de Toona ciliata cultivada em diferentes localidades. Floresta, Curitiba, PR, v. 41, n. 1, p. 47-56, 2011.

ROLIM, G. S.; SENTELHAS, P. C.; BARBIERI, V. Planilhas no ambiente EXCEL para os cálculos de balanços hídricos: normal, sequencial, de cultura e de produtividade real e potencial. Revista Brasileira de Agrometeorologia, Santa Maria, v. 6, p. 133-137, 1998.

RONDON NETO, R. M.; MACEDO, R. L. G.; TSUKAMOTO FILHO, A. A. Formação de povoamentos florestais com Tectona grandis L.f. (Teca). Boletim Técnico - Série Extensão, v. 7, n. 33, p. 1-29, 1998.

SÁ, V. A. de; MENDES, L. M.; COUTO, A. M.; LIMA, N. N. Manufatura de painéis cimento-madeira de cedro australiano (Toona ciliata M. Roem var. australis) de diferentes procedências e idade. Scientia Forestalis, Piracicaba, v. 38, n. 88, p. 559-566, 2010. 
SIQUEIRA, J. D. P.; LISBOA, R. S.; FERREIRA, A. M.; SOUZA, M. F. R. de; ARAÚJO, E. de; LISBÃO JÚNIOR, L.; SIQUEIRA, M. de M. Estudo ambiental para os programas de fomento florestal da Aracruz Celulose S.A. e extensão florestal do governo do estado do Espírito Santo. Floresta, Curitiba, Edição especial, p. 3-67, 2004.

SOUZA, J. C. A. V. de; BARROSO, D. G.; CARNEIRO, J. G. de A. Cedro australiano (Toona ciliata). Niterói: Programa Rio Rural, 2010. 12 p. (Programa Rio Rural. Manual Técnico, 21).

ThORnthwaite, C. W.; MATHER, J. R. The water balance. Publications in Climatology, 8, Centerton, New Jersey, 1955.

THORNTHWAITE, C. W. An approach toward a rational classification of climate. Geographical Review, New York, v. 38, p. 55-94, 1948.

TONELlO, K. C.; COTTA, M. K.; ALVES, R. R.; RIBEIRO, C. de F. A. POLLI, H. Q. O desenvolvimento do setor florestal brasileiro. Revista da Madeira, n. 112, 2008.

VALVERDE, S. R.; OLIVEIRA, G. G. de; SOARES, T. S.; CARVALHO, R. M. A. M. Participação do setor florestal nos indicadores socioeconômicos do estado do Espírito Santo. Revista Árvore, Viçosa, MG, v. 29, n. 1, p. 105-113, 2005. 